\title{
Modeling of Static Magnetic Anomaly Created by Iron Plates
}

\author{
Olivier Chadebec, Jean-Louis Coulomb, Vincent Leconte, Jean-Paul Bongiraud, and Gilles Cauffet
}

\begin{abstract}
This paper deals with the modeling of thin steel shells placed in a static magnetic field. The variable used is the scalar reduced potential. In front of the diversity of the formulations encountered, it proposes a methodological approach of different methods and compares them, in term of speed and easiness of computation.
\end{abstract}

Index Terms-Boundary integral method, finite elements method, magnetostatic, thin shell.

\section{INTRODUCTION}

$\mathbf{T}$ HIN Magnetic material is frequently encountered in magnetic engineering, (shielding, modeling of ships hull...). We are especially interested in the anomaly (i.e. the perturbation of the magnetic field created by these shells) when they are placed in a static external field. Classical resolutions by complete meshing of the space, frequently lead to an unacceptable increase of elements for the finite element method or to numerical instabilities for boundary element method. The formulations already developed are numerous. If some of them propose to solve the problem using the potential vector [1], we have chosen an approach with the reduced potential, because it allows separating easily the external field from the magnetic reaction of the material. This paper proposes a methodical synthesis of these formulations and a comparison between them. Advantages and drawbacks of each of them are explained, especially for integral methods.

\section{PREliminary StUdy}

The problem that we propose to solve is magnetostatic. According to Maxwell's equations,

$$
\begin{gathered}
\operatorname{div} \vec{B}=0 \\
\operatorname{curl} \vec{H}=\vec{j} \\
\vec{B}=\mu \vec{H}
\end{gathered}
$$

Let's consider the following device and notations.

Manuscript received October 25, 1999. This work was supported by French Navy (DGA)

O. Chadebec, J.-P. Bongiraud, and G. Cauffet are with the Laboratoire du Magnétisme du Navire, ENSIEG BP46, 38402 St Martin d'Hères, France (e-mail: olivier.chadebec@ @leg.ensieg.inpg.fr).

J.-L. Coulomb and V. Leconte are with the Laboratoire d'Electrotechnique de Grenoble, INPG/UJF CNRS UMR 5529, ENSIEG BP46, 38402 St Martin d'Hères, France.

Publisher Item Identifier S 0018-9464(00)05490-X.

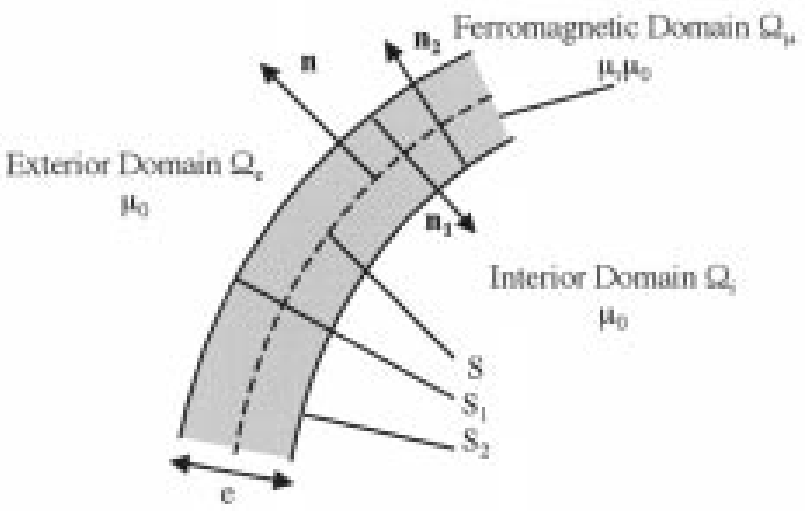

Fig. 1. Notations for the thin shell.

\section{Field in the shell}
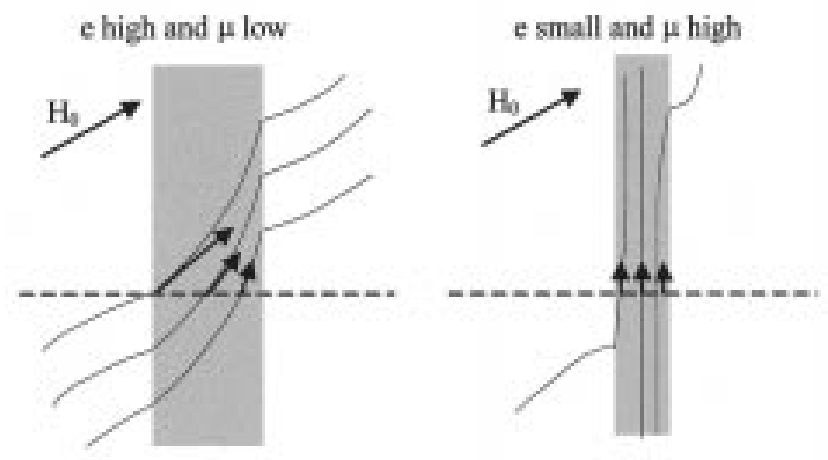

Value of the reduced potential on dotted line
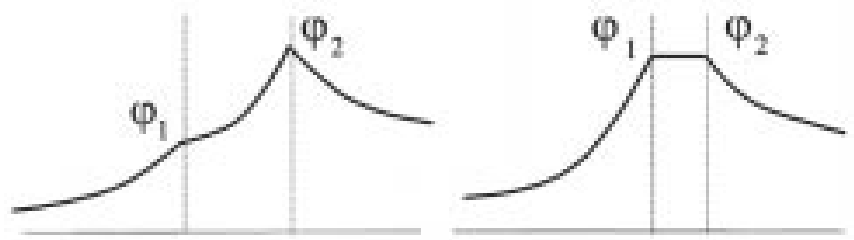

Fig. 2. Behavior of the field in the shell and associated reduced potential in function of $e$ and $\mu$.

The permeability $\mu$ is considered as constant, linear and isotropic and the shell without permanent magnetization. We suppose that the device is placed in an external field $\boldsymbol{H}_{\mathbf{0}}$ and that no current is flowing inside the ferromagnetic body.

$$
\begin{gathered}
\vec{H}=\overrightarrow{H_{0}} \overrightarrow{H_{\text {red }}} \\
\overrightarrow{H_{\text {red }}}=-\overrightarrow{\operatorname{grad} \varphi}
\end{gathered}
$$




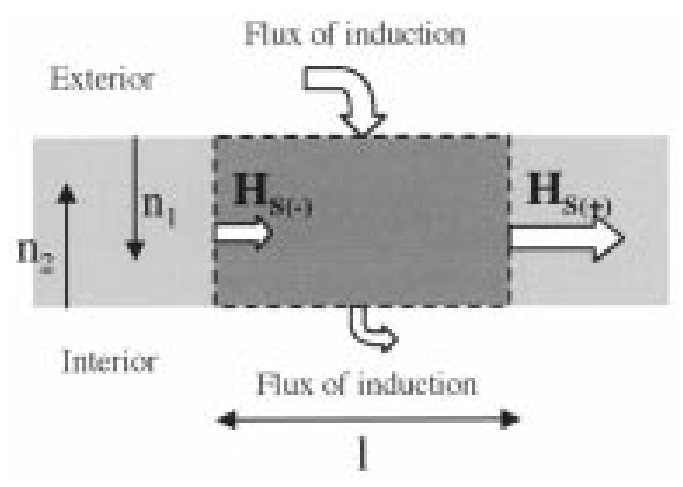

Fig. 3. Notations for the equilibrium equation of the flux in the shell.

It should be pointed out that $\boldsymbol{H}_{\text {red }}$ is the field created by the magnetic material and that this field has similar mathematical properties as an electrostatic field. Thanks to (1) and (3), the equations result into:

$$
\mu \operatorname{div}\left(\vec{H}_{0}-\operatorname{grad} \varphi\right)=0
$$

This, everywhere in $\Omega_{i}, \Omega_{\mu}$ and $\Omega_{e}$. Then, we have to solve (6) with classical continuity conditions on the two boundaries $S_{1}$ and $S_{2}$.

$$
\begin{aligned}
& H_{s_{\text {air }}}=H_{s_{\text {shell }}} \\
& B_{n_{\text {air }}}=B_{n_{\text {shell }}}
\end{aligned}
$$

That is to say in terms of reduced potential, as $\operatorname{div} \vec{H}_{0}=0$,

$$
\Delta \varphi=0
$$

with the following continuity's conditions.

$$
\begin{gathered}
\varphi_{\text {air }}=\varphi_{\text {shell }} \\
-\mu_{0} \frac{\partial \varphi_{\text {air }}}{\partial n}+\mu \frac{\partial \varphi_{\text {shell }}}{\partial n}=\left(\mu-\mu_{0}\right) H_{0 n}
\end{gathered}
$$

\section{BEHAVIOR OF THE SHEET}

The main idea of this part is to find an equation describing the physical behavior of the sheet. We now consider that the permeability of the sheet is high in comparison to the permeability of the air and that the thickness $e$ is small in comparison to other dimensions. The higher the permeability is, the better the flux is driven inside the sheet. The field in the sheet is mainly tangential and constant throughout the thickness of the shell. If the variation of $H_{0 n}$ is negligible, the reduced potential is the same on both sides of the sheet.

Fig. 3 describes the behavior of the flux going inside and outside a small steel element.

The dimension $l$ is small enough to consider the induction being constant along the boundary between shell and air. Moreover, the permeability being high, the induction is assumed normal on this boundary. An equilibrium equation between flux can be written.

$$
e\left(\mu H_{S}(-)-\mu H_{S}(+)\right)=l\left(-B_{n_{1}}-B_{n_{2}}\right)
$$

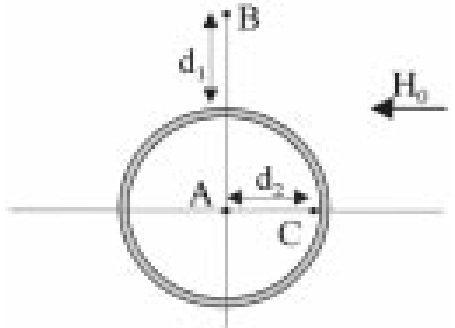

Fig. 4. Notations for the infinite cylinder. $\mu_{r}=1000, e=1 \mathrm{~mm}, R=1 \mathrm{~m}$, $H_{0}=100 \mathrm{~A} / \mathrm{m}, d_{1}=1 \mathrm{~m}, d_{2}=0.97 \mathrm{~m}$.

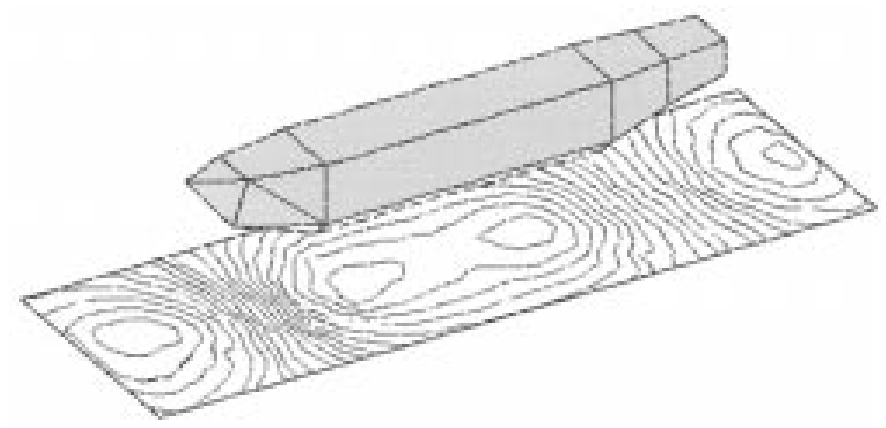

Fig. 5. Modeling of a mock-up of a ship ( $4.6 \mathrm{~m}$ length) placed in a longitudinal external field by integral local formulation (BEM/FEM). $e=1.4 \mathrm{~mm}, \mu_{r}=$ $96, H_{0}=1000 \mathrm{nT}$, Modulus of the field on a reference plane $(30 \mathrm{~cm}$ under the hull).

If $l$ tends toward a zero limit, we obtain, by combining (12), (4) and (5) and assuming that $\boldsymbol{n}=-\boldsymbol{n}_{\mathbf{1}}=\boldsymbol{n}_{\mathbf{2}}$,

$$
\operatorname{ediv}_{s}\left(\mu \vec{H}_{s}\right)=\mu_{0}\left(-\frac{\partial \varphi_{1}}{\partial n}+\frac{\partial \varphi_{2}}{\partial n}\right)
$$

where $\operatorname{div}_{s}$ is the standard divergence applied only on the tangential components of the field. In this way, we get surface partial differential equation. To reduce the shell to a surface is an approximation. The volume of air is then artificially increased and the energy in it will be too high. A good solution is to replace in (13), the field in the shell $\boldsymbol{H}_{\boldsymbol{s}}$ by $\boldsymbol{H}_{\boldsymbol{s}}$ minus by the value that it will have in the air [2]. Then, we obtain:

$$
\operatorname{ediv}_{s}\left(\mu \vec{H}_{s}-\mu_{0} \vec{H}_{s}\right)=\mu_{0}\left(-\frac{\partial \varphi_{1}}{\partial n}+\frac{\partial \varphi_{2}}{\partial n}\right)
$$

Equation (14) will allow us to model devices with smaller permeability value with more accuracy. As $\mu$ is isotropic and constant, we have:

$$
e\left(\mu_{r}-1\right) \operatorname{div}_{s}\left(\overrightarrow{H_{0_{s}}}-g r \overrightarrow{a d} d_{s} \varphi\right)=-\frac{\partial \varphi_{1}}{\partial n}+\frac{\partial \varphi_{2}}{\partial n}
$$

where $\operatorname{grad}_{s}$ is the standard gradient applied only on the tangential components of $S$.

\section{LOCAL FORMULATIONS}

These formulations allow separating the domains and solving the different equations on each of them. Two domains $\Omega_{i}$ and $\Omega_{e}$ are ruled by (6) and while (15) applies to surface $S$. A local system of equations is obtained for each region. Interface conditions link these systems. 


\section{A. Modeling of the Sheet}

We build $N$ Galerkin weighting functions $W_{i}$ on the surface $S$, the potential is approximated by:

$$
\varphi=\sum_{i=1}^{N} W_{i} \varphi_{i}
$$

The Galerkin weighted residual method applied on (15) provides a system of $N$ equations.

$$
\begin{aligned}
& \sum_{j=1}^{N} \varphi_{j} \iint_{S} k \operatorname{grad}_{S} W_{i} \cdot \operatorname{grad}_{S} W_{j} d s \\
& \quad=\iint_{S} k \operatorname{grad}_{S} W_{i} \cdot \vec{H}_{0} d s-\iint_{S}\left(\frac{\partial \varphi_{j 2}}{\partial n}-\frac{\partial \varphi_{j 1}}{\partial n}\right) W_{i} d s
\end{aligned}
$$

where $k=e\left(\mu \backslash \mu_{0}-1\right)$ and $i=1, \cdots, N$.

\section{B. Modeling of the Air}

1) Finite Element Method: The same procedure as above, but for $M$ Galerkin weighting functions built in the air region gives:

$$
\begin{aligned}
& \sum_{j=1}^{M} \varphi_{j} \iiint_{\Omega_{i}+\Omega_{e}} \operatorname{grad} W_{i} \operatorname{grad} W_{j} d v \\
& \quad=\iiint_{\Omega_{i}+\Omega_{e}} \operatorname{grad} W_{i} \cdot \overrightarrow{H_{0}} d v-\iint_{S}\left(\frac{\partial \varphi_{j 2}}{\partial n}-\frac{\partial \varphi_{j 1}}{\partial n}\right) W_{i} d s
\end{aligned}
$$

Main difficulty of this method is that the domain is infinite. It is necessary to develop a special numerical tool, which takes in account the infinite region by mapping it into a closed domain [3]. By combining this system with (17), we obtain a sparse matrix where the unknowns are the reduced potential on each node. Interface conditions are such as the terms integrated on $S$ disappear.

2) Boundary Element Method: Potential being constant on the shell, according to Green's theorem, two surface equations can be written [4],

$$
\begin{gathered}
c_{1} \varphi=-\int_{S}\left(\varphi \frac{\partial G}{\partial n}-G \frac{\partial \varphi_{1}}{\partial n}\right) d \Gamma \\
c_{2} \varphi=\int_{S}\left(\varphi \frac{\partial G}{\partial n}-G \frac{\partial \varphi_{2}}{\partial n}\right) d \Gamma
\end{gathered}
$$

where $G$ is the $1 / r$ Green's function and $c$ the Cauchy principal value. A point collocation method can be applied on (19) and (20). If $S$ is meshed in $N$ elements, (19) becomes:

$$
c_{i 1} \varphi_{i}+\sum_{j=1}^{N} \varphi_{j} \iint_{S_{j}} \frac{\partial G_{i j}}{\partial n} d s_{j}=\sum_{j=1}^{N}-\frac{\partial \varphi_{1 j}}{\partial n} \iint_{S_{j}} G_{i j} d s_{j}
$$

We have to solve these two systems with (15). The unknowns are the reduced potential on the shell and the normal derivative on each sides of the sheet. Field at the infinity is taken in account by the Cauchy principal value $c_{i 1}$.

\section{GLOBAL FORMULATIONS}

As we have said, the magnetic anomaly has the mathematical properties of an electrostatic field. The idea leading to global formulation is to suppress the magnetized material and to replace it by a charge distribution located on $S$, which creates the same potential in the air. Equation (15) can be also interpreted as a local form of the Gauss theorem, where the source of the anomaly is a surface charge density $q$, which has the following value:

$$
q=\frac{\partial \varphi_{1}}{\partial n}-\frac{\partial \varphi_{2}}{\partial n}
$$

Equation (15) becomes:

$$
e\left(\mu_{r}-1\right) \operatorname{div}_{s} \vec{H}_{s}=-q
$$

The reduced potential will thus be expressed with the following single layer distribution.

$$
\varphi=\int_{S} q G d \Gamma
$$

Magnetization can also be represented by a volume distribution of dipoles. As the magnetization is constant through the shell, we can replace it by a surface distribution of dipoles. Its surface density is equivalent to a surface density of charges and they are linked by [5]:

$$
\operatorname{div} \overrightarrow{p_{s}}=-q
$$

Equation (23) is then equivalent to:

$$
e\left(\mu_{r}-1\right) \overrightarrow{H_{S}}=\overrightarrow{p_{s}}
$$

The reduced potential will be expressed with the help of a double layer distribution expressed by:

$$
\varphi=\int_{S} \overrightarrow{p_{s}} \cdot \operatorname{grad} G d \Gamma
$$

\section{A. Global Variational Formulation}

A constant charge density $q_{i}$ is on each element of the sheet. Equation (24) and a point collocation method give the following system:

$$
\varphi_{i}=\sum_{j=1}^{N} q_{j} \iint_{T j} G_{i j} d s j
$$

We need to inverse this system to express $q$ as a function of $\varphi$, which leads to solve $N$ systems of equations. The matrix of $q$ is combined with (17) to obtain a system in $\varphi$. Once it is solved, it's easy to obtain $q$ with (28) and then the value of the potential everywhere using (24) [6].

\section{B. Global Equation of Surface Flux Equilibrium}

Let's consider each element carrying a punctual constant total charge $Q_{i}$. Let's apply (23) on an element $S_{i}$ and apply the Ostrogradsky's theorem reduced to its surface form. We get:

$$
-Q_{i}=e\left(\mu_{r}-1\right) \int_{l_{i}}\left(\overrightarrow{H_{0_{s}}}-\operatorname{grad}_{s} \varphi\right) \cdot \overrightarrow{n_{l}} d l_{i}
$$


where $l_{i}$ is the contour of each element $i$ and $n_{l}$ a vector tangential to $\Gamma$ and orthogonal to $l_{i}$. Equation (28) and (29) leads to:

$$
-Q_{i}+\sum_{j=1}^{N} Q_{j} \int_{l j} k \cdot \operatorname{grad}_{S} G_{i j} \cdot \vec{n}_{l} d l_{j}=\int_{l j} k \cdot \vec{H}_{0} \vec{n}_{l} d l_{j}
$$

Main interest of this formulation is that the surface integrals become line integrals. Moreover, analytical integration is possible. The number of unknowns is $N$. However, integration of the source's term is a difficulty and requires the generation of nonplane elements [7]. If all the elements are plane the last term in (30) vanishes and the solution found is zero for each $Q_{i}$.

Using the field of punctual dipoles can perform a similar dipole formulation. The expression of the potential becomes:

$$
\varphi_{i}=\sum_{j=1}^{N} \overrightarrow{P_{j}} \iint_{S_{j}} \operatorname{grad} G_{i j} d S_{j}
$$

Let's integrate (26) on an element $s_{i}$ and apply the Ostrogradsky's theorem reduced to its surface form. We get:

$$
\overrightarrow{P_{i}}-\sum_{j=1}^{N} Q_{j} \int_{l j} k \cdot \operatorname{grad}_{S} G_{i j} \cdot \overrightarrow{n_{l}} d l_{j}=\int_{S j} \overrightarrow{H_{0_{S}}} d S_{j}
$$

This equation is vectorial, the number of unknowns is then $2 \times N$ (the vectors are tangential to $S$ ). The integration of the source term can be done there on plane elements. For all these methods, the systems obtained are full.

\section{NUMERICAL RESULTS}

The FEM being well known, we were especially interested by the integral formulations.

\section{A. 2D Modeling}

To compare numerical accuracy of these global methods, we consider an infinite cylinder placed in an homogenous external field. This $2 \mathrm{D}$ problem can be solved analytically. In the variational approach, the charges are taken constant on each element and, in the flux equilibrium formulations, punctual. All integrations are analytical. The mesh used is coarse (36 elements). The different points of calculation are the followings:

These integral formulations lead to very efficient results for most points of calculation. Charge formulation provides the best accuracy. Let's notice that the dipole one leads to divergence problems if the point is too close to the device (the increase of the potential being more divergent at the neighborhood of a double layer distribution than at the neighborhood of a single one).

\section{B. 3D Modeling}

We are especially interested in computing anomaly created by the steel hull of ships. Some of these formulations have been developed in $3 \mathrm{D}$.

\section{CONCLUSION}

As conclusion, we propose a comparison of these formulations. Our criteria for time of computation will be the size of
TABLE I

Field CAlCulation for the DifFerent POINTS

\begin{tabular}{cccc}
\hline Formulations & Point A & Point B & Point C \\
\hline Analytic & 66.744 & 91.664 & 66.744 \\
Variational & 66.597 & 91.689 & 72.320 \\
Charges & 66.724 & 91.681 & 88.562 \\
Dipoles & 66.593 & 91.681 & 71.731 \\
\hline
\end{tabular}

\begin{tabular}{|c|c|c|c|c|}
\hline \multirow[b]{2}{*}{ Designation } & \multirow{2}{*}{$\begin{array}{c}\begin{array}{c}\text { Local } \\
\text { formulation }\end{array} \\
\text { FEM/BEM }\end{array}$} & \multicolumn{3}{|c|}{ Global formulation } \\
\hline & & Variational & Charges & Dipoles \\
\hline Unknowns & $\varphi_{2} \frac{\partial \varphi_{1}}{\partial n}, \frac{\partial \varphi_{2}}{\partial n}$ & $q$ then $\varphi$ & Q & $\overrightarrow{\mathrm{P}}$ \\
\hline $\begin{array}{l}\text { Size of } \\
\text { systems to } \\
\text { solve }\end{array}$ & $\begin{array}{c}\text { one of } \\
(3 \times N)^{2}\end{array}$ & $\frac{(N+1)}{N^{2}}$ of & $\begin{array}{c}\text { one of } \\
\mathrm{N}^{2}\end{array}$ & $\begin{array}{l}\text { one of } \\
(2 \times N)^{2}\end{array}$ \\
\hline Integration & Surface & Surface & Line & $\begin{array}{l}\text { Line \& } \\
\text { Surface }\end{array}$ \\
\hline $\begin{array}{c}\text { Plane } \\
\text { elements }\end{array}$ & Yes & Yes & No & Yes \\
\hline
\end{tabular}

TABLE II

COMPARISON OF THE DIFFERENT BEM

the obtained system because it is significant of both times of building and resolution. Charges method is the best (one system of $N \times N)$, followed by the dipole $(2 N \times 2 N)$ and then the boundary local formulation $(3 N \times 3 N)$. The variational one is the most expensive in terms of CPU-times (one inversion of a $N \times N$ matrix plus one $N \times N$ system to solve). In term of easiness of computation, our criteria will be the generation of the mesh; this is the most important drawback of charge formulation. It's necessary to generate some nonplane elements [7]. The choice of a formulation depends essentially of the geometry of the device. For a 2D modeling, the most suitable solution is the dipole one. Their direction being restricted to the plane, the system becomes $N \times N$. For 3D modeling, the choice is more complex. To generate the charge mesh can be long and laborious but the matrix obtained (which is full) keeps a reasonable size for a good numerical accuracy. For other formulations, these sizes increase very quickly (see Table II). All these methods are easy to implement with variable thickness. Moreover, it's possible to take into account permanent magnetization for the sheet (except in the charge formulation).

\section{REFERENCES}

[1] H. Igarashi and T. Honma, "An analysis of thin magnetic materials using hypersingular integral equations," IEEE Trans. Magn., vol. 32, pp. 682-685, May 1996.

[2] X. Brunotte and G. Meunier, "Line element for efficient computation of the magnetic field created by thin iron plates," IEEE Trans. Magn., vol. 26, pp. 2196-2198, September 1990. 
[3] X. Brunotte, G. Meunier, and J. F. Imhoff, "Finite elements solution of unbounded problems using transformations: A rigorous, powerful and easy solution," IEEE Trans. Magn., vol. 28, pp. 1663-1666, March 1992.

[4] L. Krahenbuhl and D. Muller, "Thin layer in electrical engineering. Example of shell models in analyzing eddy-currents by boundary and finite element methods," IEEE Trans. Magn., vol. 29, pp. 1450-1455, March 1993.
[5] E. Durand, Magnétostatique Masson et Cie, Paris, 1968.

[6] F. Rioux-Daminau, B. Bandelier, and P. Penven, "A fast and precise determination of the static magnetic field in the presence of thin iron shells," IEEE Trans. Magn., vol. 31, pp. 3491-3493, November 1995.

[7] A. Vishnevski, I. Krasnov, and A. Lapokov, "Calculation of static magnetization for thin-walled constructions by boundary element method," IEEE Trans. Magn, vol. 29, pp. 2152-2155, September 1993. 